Raul Philipe Marcos Silva ${ }^{1}$ Cynthia Maria Barboza do Nascimento ${ }^{1}$ (16)

Gabriella Morais Duarte Miranda² Vanessa Lima da Silva ${ }^{3}$ Maria Luiza Lopes Timóteo de Lima4 ${ }^{4}$

Mirella Bezerra Rodrigues Vilela ${ }^{1}$

\section{ERRATA: "Evolução da oferta de Fonoaudiólogos no SUS: um estudo sobre a correlação com os indicadores sociais no Brasil na última década"}

No artigo intitulado "Evolução da oferta de Fonoaudiólogos no SUS: um estudo sobre a correlação com os indicadores sociais no Brasil na última década", com o número do DOI: https://doi.org/10.1590/2317-1782/20202019243, publicado na revista CoDAS, 2021, volume 33, número 2, na página 1:

Onde se lia:

"Vanessa Lima da Silva ${ }^{3}$ http://orcid.org/0000-0002-0548-9664

Leia-se:

"Vanessa de Lima Silva ${ }^{3}$ http://orcid.org/0000-0002-1562-1761

\footnotetext{
${ }^{1}$ Departamento de Fonoaudiologia, Universidade Federal de Pernambuco - UFPE - Recife (PE), Brasil.

${ }^{2}$ Área Acadêmica de Medicina Social e Programa de Pós-graduação em Saúde Coletiva, Universidade Federal de Pernambuco - UFPE - Recife (PE), Brasil.

${ }^{3}$ Departamento de Fonoaudiologia e Programa de Pós-graduação em Gerontologia, Universidade Federal de Pernambuco - UFPE - Recife (PE), Brasil.

${ }^{4}$ Departamento de Fonoaudiologia e Programa de Pós-graduação em Saúde da comunicação Humana, Universidade Federal de Pernambuco - UFPE - Recife (PE), Brasil.
} 